\title{
ANALYSIS BY FINITE ELEMENT METHOD OF THE CROSSTALK ERRORS ASSOCIATED TO THE GEOMETRICAL PARAMETERS FOR CURRENT TRANSDUCERS.
}

\author{
Arcega F.J., Artal J.S., Letosa J. and Usón A. \\ Department of Electrical Engineering. Escuela Universitaria de Ingeniería Técnica Industrial. \\ University of Zaragoza, Campus Rio Ebro. María de Luna n ${ }^{\circ} 3$. \\ Edificio Torres Quevedo, 50018. Zaragoza, Spain. \\ E-mail:arcegafj@unizar.es,jsartal@unizar.es,jletosa@unizar.es,andauson@unizar.es
}

\begin{abstract}
Keywords.
High current measurement; Geometrical parameters; Stable point; current transducers; Finite Element Method.
\end{abstract}

\section{Introduction.}

The aim of this work is the study of the influence of geometrical parameters associated to a model of current sensor in the cross-talk error when measuring high electrical currents. In a previous paper [1] the existence of a point -stable point- where the influence of the current circulating by the external conductor in the measurement of the internal current was null, was analysed. In this point was no influence the sense of the current or his value. This work shown was developed in a model with dimensions of conductors and predetermined geometrical parameters because only the authors wanted to analyse the existence of this point. The core used was a commercial non-linear iron powder toroidal core (yellow-white) ref. T300$26 \mathrm{D}$.

In [2] a comparison between this method -stable pointand other two systems of current measurements, sensor arrays [3] and linear equations [4], showing advantages and disadvantages of those measurement methods and the cross-talk errors associated was carried out.

In this paper we try to advance a bit more. We will analyse the geometrical dependence (section of conductors, distance between them, permeability, ...) of this -stable point- with the aim of validate our model and observe the compensation of cross-talk errors originated by the current carried by near conductors [5], [6] under normal working conditions
[7]. In this way, a comparison between the uncertainties associated to the geometrical parameters -values numerically calculated with the modelization by Finite Element Method- and the magnetic flux of an infinite straight line calculated theoretically by the Biot-Savart law has been done. Simulations have been developed using the commercial software OPERA-3D of Vector Fields for low frequency fields $(50 \mathrm{~Hz})$. All these situations have been simulated by the Finite Element Method (FEM) and have been measured and verified in our Laboratory of Electrical Metrology (LME) accredited by ENAC, the Spanish Accreditation System in the frame of the EA (European Accreditation).

\section{Influence of geometrical parameters.}

The analysed system consists, see figures 1 and 2, of a single circuit with currents flowing through two straight conductors of normalised rectangular section $\left(a \times b \mathrm{~mm}^{2}\right)$. One of the bars is placed in the centre of the measuring toroidal core, where the sensors are positioned. The two parallel conductors have the same geometry and the perpendicular distance between them is $d \mathrm{~mm}$. The geometrical characteristics of the core are the following: inner and outer radius $R_{I N}=25 \mathrm{~mm}, R_{E X}=39 \mathrm{~mm}$ and height $h=25,4 \mathrm{~mm}$. The core used has a relative permeability $\mu$. Magnetic flux through a cross-section of the torus was chosen as the result from the numerical calculations as the response of a current transducer and measurement errors were evaluated.

Figure 2 show -in color contour map format- the influence of a near external current $\left(I_{C E X}=300 A\right)$ in the magnetic flux in the inner of the torus of permeability $\mu=1$ (where the sensors are situated) originated by an internal current $\left(I_{C I N}=300 A\right)$. The 
dimensions of the conductors are $a=40 \mathrm{~mm}, b=5 \mathrm{~mm}$ and the distance between both is $d=50 \mathrm{~mm}$. The computer simulations by finite element method (FEM) have allowed to estimate the errors associated to the magnetic flux in each studied method with respect to the ideal situation, analyzing in all cases the influence of the current in the measuring system. In this way, the errors associated to the computational analysis have been estimated under $\Delta \varepsilon_{F E M}=+0,18 \%$ (number nodes $=448473$, number equations $=427896$ ). Another possible source of error in the measurement comes from the uncertainty in the positioning of the sensors around the conductor: small variations with respect to the suitable angle give rise to non negligible errors in the different methods of measurement based on numerical compensation.

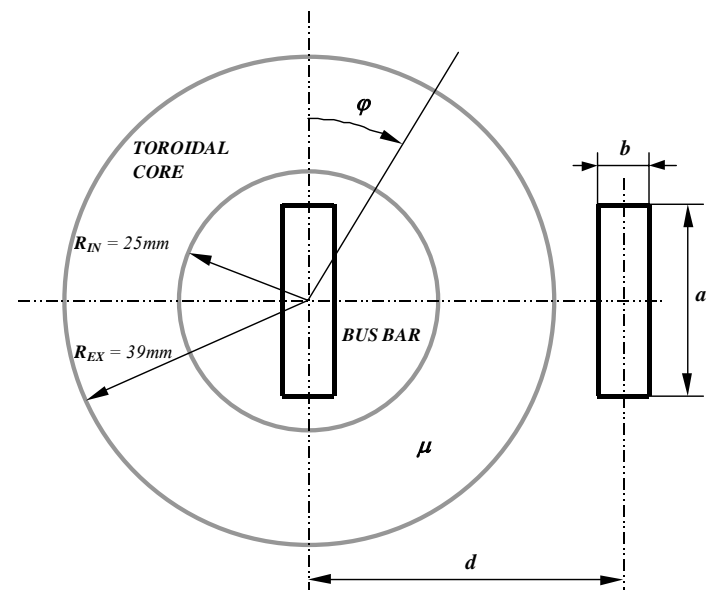

Fig 1. Illustration of the experimental model with the parameters of the rectangular conductor $a \times b$ and the distance $d$.

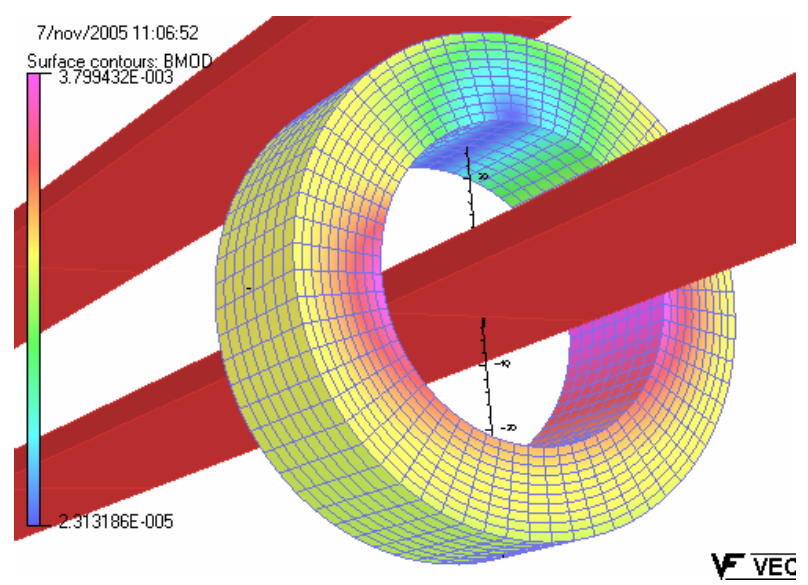

Fig 2. Influence or current near to conductor, simulation of the single circuit model with two conductor, $\mu=1 ; I_{C I N}=I_{C E X}=300 \mathrm{~A} ; d=50 \mathrm{~mm}$.

The figure 3 shows the error produced in the magnetic flux (influence of cross-talk) with respect to the angular position of the sensor and in function of the permeability $\mu$ of the toroid. In all the analysed cases, the currents have been maintained constant $I_{C I N}=300 \mathrm{~A}, I_{C E X}=300 \mathrm{~A}$ and as well the parameters associated to the rectangular conductors $a=40 \mathrm{~mm}$, $b=5 \mathrm{~mm}$ and the distance $d=50 \mathrm{~mm}$. It can be seen that when the permeability of the torus raises the cross-talk error tends to be independent of the angular position of the sensor. This flux error is maintained constant (see curve of $\mu=7500$ ) so when $\varphi=0^{\circ} \rightarrow \Delta \varepsilon=-0,372 \%$. Apparently is possible to see a point of cut in the curves of permeability $\mu \neq \mu_{O}$, point that correspond with the angular position $\varphi=15,305^{\circ} \rightarrow \Delta \varepsilon=-0,392 \%$ and where the influence of the permeability of the toroidal core in the measurement will be null.

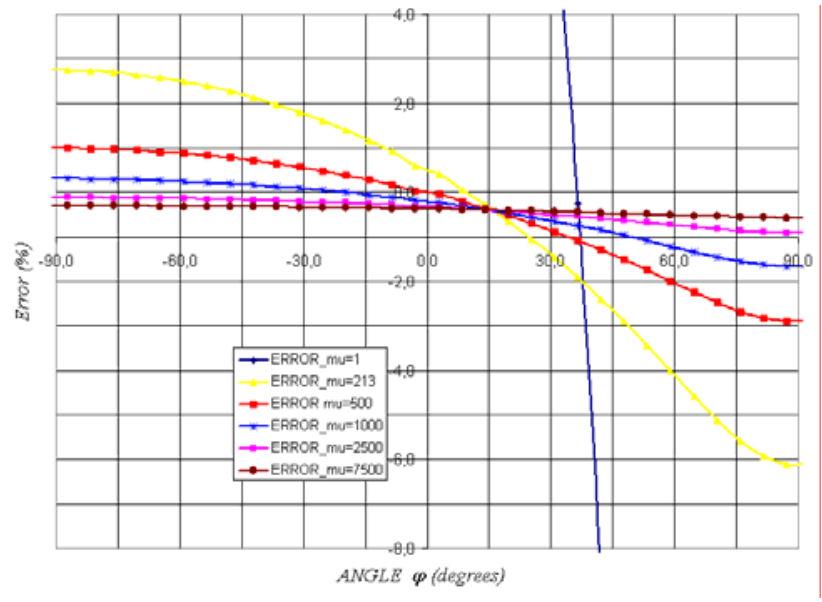

Fig 3. Magnetic flux (crosstalk effect) vs. sensor angular position in the core for several permeability values $I_{C I N}=I_{C E X}=300 \mathrm{~A} ; d=50 \mathrm{~mm}$.

As can be seen in the figure, the case of the permeability $\mu=\mu_{O}$ is not considered due to the high margin of deviation that present in the measurement $-142,943 \% \leq \Delta \varepsilon \leq+40,330 \%$. As well is possible to see the angle where the error is null -stable point- [1] that correspond with $\varphi=+36,25^{\circ}$. In this position, with a small angular displacement of $\Delta \varphi= \pm 1,0^{\circ}$, a margin of variation in the flux measurement of $+1,294 \% \leq \Delta \varepsilon \leq-1,287 \%$ will be obtained. It shows the great sensibility of the model with respect to the errors of angular positioning, because of the great slope that presents the curve $\mu=1$ in the places near the stable point, associated to the construction process and that produces that the use with air was unviable.

After obtaining the graph corresponding to the case of linear permeability, shown in the figure 3, we have analysed the response of the material of the toroyd considering that it is made of an isotropic and nonlinear permeability material. For this case, the characteristic curve of the material has been used $B=f(H)$ and the behaviour for the FEM has been analysed in the monophasic case (for both conductors circulate the same current $I_{C I N}=I_{C E X}$ 
The figure 4 shows a comparison between the error produced with respect to the angular position of the transductor in function of the current carried by both conductors in the case of two cores composed by different materials NETIC S3-6 -alloy from Magnetic Shield Corporation effectively shields extremely high magnetic fields- and LOSIL1000-65 -laminated steel sheet manufacturer's curve.

It can be seen that in the figure 4 appear the point analysed previously, independent of the type of material in the core of the toroyd. This point correspond, in the case of the LOSIL1000-65 with the angular position $\varphi=+18,74^{\circ} \pm 1^{\circ}$.

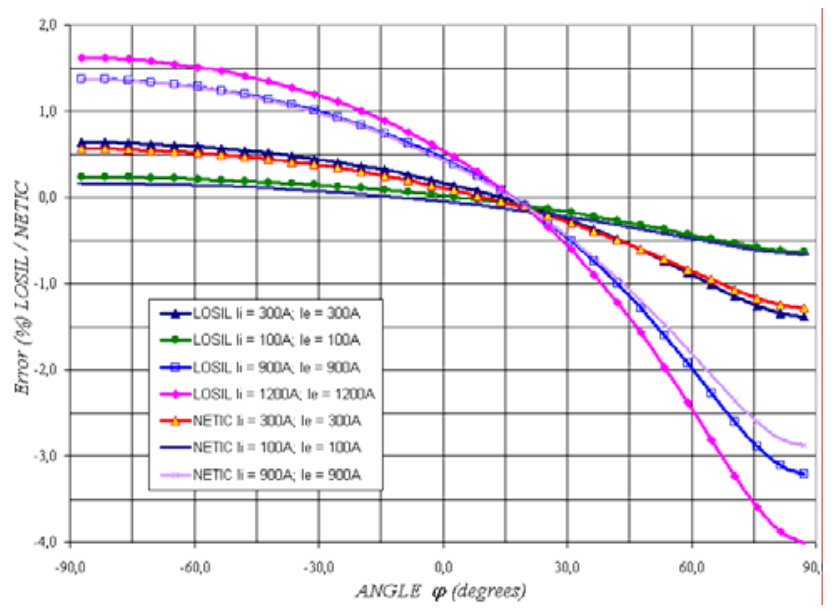

Fig 4. Magnetic flux error vs. sensor angular position in the core for two material types, where $\mu$ is nonlinear curve and $I_{C I N}=I_{C E X}$.

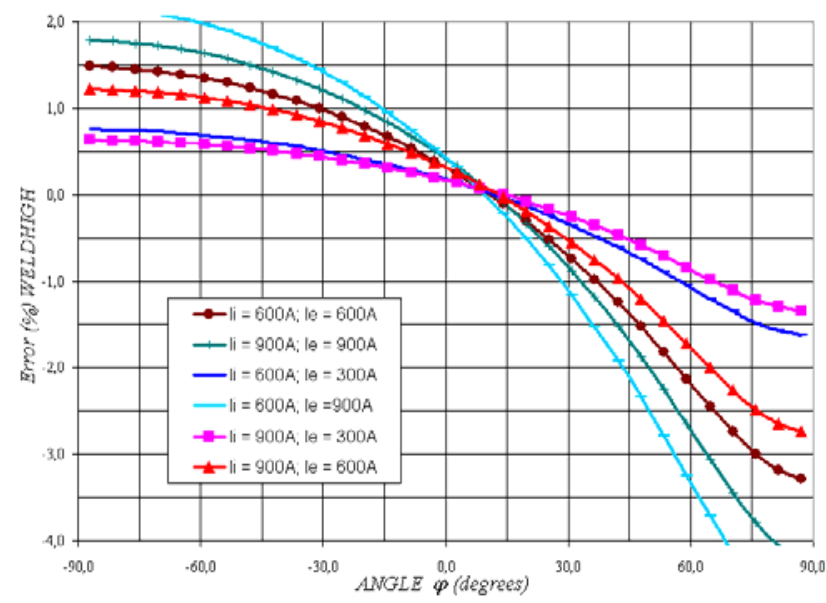

Fig 5. Magnetic flux error vs. sensor angular position for a core from weld high material, where $\mu$ is nonlinear curve and $I_{C I N} \neq I_{C E X}$.

In reference [1] it is described the existence of an angular position in the toroydal core where the influence of the external electric current in the measurement of magnetic flux, proportional to the current in the inner conductor, is nearly zero -stable point- Moreover it was shown that such angular position $\varphi$ depends, between other factors, of the magnetic permeability of the used core. As a complement, in the figure 3 can be seen that the values of maximal deviation in the magnetic flux diminish when the value of the magnetic permeability increases. In this way, the figure 5 show the stable point and the light displacement in function of the current of the inner conductor because of the change of the permeability of the used core $B=f(H)$.

In the next example an isotropic core with non-linear permeability constituted by WELDHIGH -Upper quartile curve measured in weld material a weld and mild steel plate. Source of data: The Welding Institute 1/Dec/1986-.has been considered. In such case, for a current of $I_{C I N}=600 \mathrm{~A}$, the stable point correspond to an approximate position of $\varphi=+16,34^{\circ} \pm 1,03^{\circ}$, while for a current of $I_{C I N}=900^{a}$ the new stable point is placed in $\varphi=+18,54^{\circ} \pm 1,2^{\circ}$. This slight variation in the angular position can be covered by the size of the sensor used

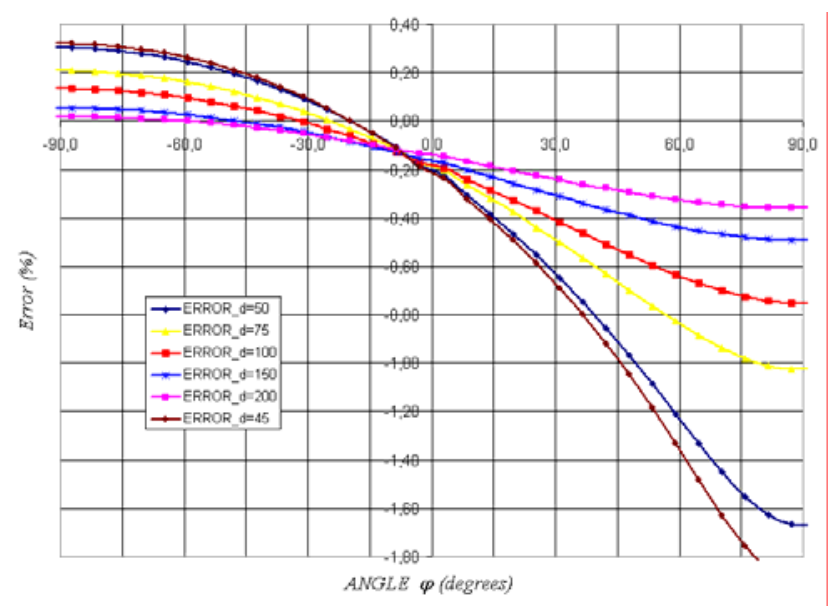

Fig 6. Magnetic flux error vs. sensor angular position in the core for several distance values " $d$ " $I_{C I N}=I_{C E X}=300 \mathrm{~A} ; \mu=1000$.

The figure 6 shows the error produced in the magnetic flux (cross-talk effect) with respect to the angular position of the sensor and in function of the parameter $d$-distance between conductors-. In the tested cases the currents have been maintained constant $I_{C I N}=300 \mathrm{~A}, I_{C E X}=300 \mathrm{~A}$, the dimension of conductors $a=40 \mathrm{~mm}, b=5 \mathrm{~mm}$ and the permeability of the toroid $\mu=1000$. As could be expected, when the external conductor is separated, the error in the measurement of the internal current diminishes. So for having errors lower than $\Delta \varepsilon= \pm 0,2 \%$ with a distance between conductors $d=200 \mathrm{~mm}$ it will be enough to place the sensor between the angles $\Delta \varphi \leq+18,146^{\circ}$, while for a distance $d=50 \mathrm{~mm}$ and for the same error, we will need to place the sensor in a position between the angles $-48,616^{\circ} \leq \Delta \varphi \leq+0,232^{\circ}$. Observing the figure is possible to see a point where the curves cut together 
that corresponds where the parameter $d$ does not affect to the measurement, angle that corresponds to $\varphi=-5,921^{\circ} \rightarrow \Delta \varepsilon=-0,140 \%$.

After analysing the effects of the permeability in the core and the distance of the external conductor, now we will analyse the geometrical parameters of the rectangular internal conductor of section $a \times b \mathrm{~mm}^{2}$. For that we will compare the flux obtained in the different cases (result of the modification of the section of the conductor) with the values of the flux in the ideal case. In all the studied cases, the current circulating by the internal conductor is $I_{C I N}=300 \mathrm{~A}$ and the relative permeability of the core $\mu=1000$. The ideal magnetic flux corresponds to the one obtained theoretically for an infinite straight cable and for the data of our problem gives a value of $693,70 \mu \mathrm{Wb}$.

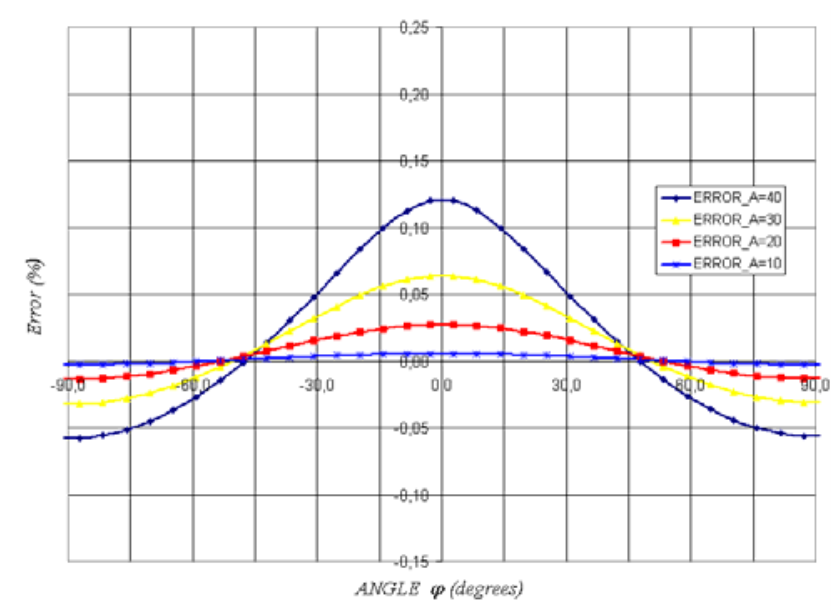

Fig 7. Magnetic flux error vs. sensor angular position in the core for several width values $I_{C I N}=300 \mathrm{~A}$; $\mu=1000 ; b=5 \mathrm{~mm}$.

The figure 7 shows the error produced in the magnetic flux with respect to the angular position of the sensor and originated by the use of a rectangular conductor of width $a$. In the tested cases, the current has been maintained constant $I_{C I N}=300 \mathrm{~A}$, as well the thickness of the conductor $b=5 \mathrm{~mm}$ and the permeability of the core $\mu=1000$. It is verified that further the ideal case (increment of the parameter $a$ approximating to the internal radius of the toroidal core $R_{I N}=25 \mathrm{~mm}$ ) an increase of the error is produced. The maximum deviation with respect to the ideal case is produced when angular position $\varphi=0^{\circ}$ being the most unfavourable case, $a=40 \mathrm{~mm}$, inferior to $-0,057 \% \leq \Delta \varepsilon \leq+0,119 \%$.

In the same way, the figure 8 shows the error produced in the magnetic flux with respect to the angular position of the sensor by using a rectangular conductor of thickness $b$. In the tested cases the current has been maintained constant $I_{C I N}=300 \mathrm{~A}$, as well the width of the conductor $a=40 \mathrm{~mm}$ and the permeability of the torus $\mu=1000$, (the case of width more unfavourable has been taken for observing the influence in the error of measurement for the increment of the thickness of the conductor). In the figure can be checked that when the width of the rectangular conductor diminishes $b=2,5 \mathrm{~mm}$ the error in the measurement of flux increases slightly $\varphi=0^{\circ} \rightarrow \Delta \varepsilon=+0,122 \%$. While the increase of the parameter $b$ supposes a diminution of the deviation with respect to the ideal case, for $b=15 \mathrm{~mm}$ the error will be between $-0,050 \% \leq \Delta \varepsilon \leq+0,098 \%$.

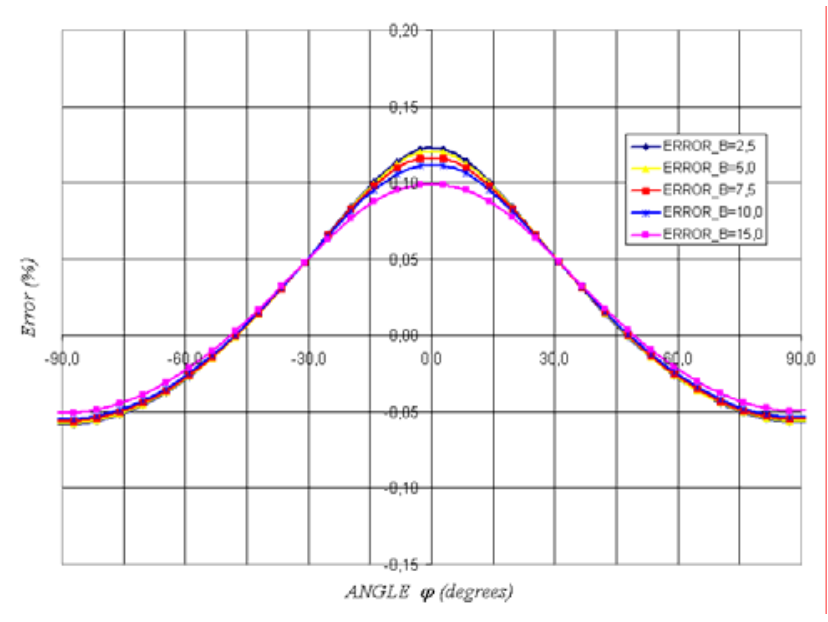

Fig 8. Magnetic flux error vs. sensor angular position in the core for several thickness values $I_{C I N}=300 \mathrm{~A}$; $\mu=1000 ; a=40 \mathrm{~mm}$.

As it will be logical changes in the geometrical parameters of the section of the internal conductor $a \times b$ does not produce a deviation so important with respect to the ideal case as the relative permeability $\mu$ of the toroidal core used in the model or the distance $d$ of the external conductor. The contribution to the error of the measurement produced by the non centred conductor in the torus has not been considered here because it was studied in [7] for a similar case.

\section{Conclusions.}

The present study contributes to improve the knowledge about the uncertainty sources in current measurements based on sensor or transducers. Furthermore, it explores the possibilities of using FEM simulations to reduce the experimental arrangements needed for calibrating these devices. Consequently this work is devoted to help the metrology authorities and instrumentation manufacturers to develop new rules for calibration devices and new procedures to characterize the precision of the equipment.

The computer simulations by FEM have allowed to estimate the errors associated to the magnetic flux in each studied method with respect to the ideal situation, 
analyzing in all cases the influence of the current in the measuring system.

\section{Acknowledgements.}

This paper has been developed with the financial support of the University of Zaragoza (Program 200102), Ibercaja (Research Program 2003) and the Diputación General de Aragón (Emergent Research Group 2004/2005).

\section{$\underline{\text { References }}$}

[1]. M. Samplón, J.S. Artal, J. Letosa, A. Usón and F.J. Arcega "Evaluation of Errors associated with Crosstalk Magnetic Fields using Finite Element Method in High Electrical Current Measurement". Internacional Conference on Renewable Energy and Power Quality, ICREPQ 2005. Zaragoza, Spain. March 2005.

[2]. A. Usón, J.S. Artal, J. Letosa, F.J. Arcega and M. Samplón "Analysis by Finite Element method of the Crosstalk Compensation procedures for Current Transducers". Internacional Symposium on Electromagnetic Fields in Mechatronics, Electrical and
Electronic Engineering, ISEF 2005. Baiona, Spain. September 2005.

[3]. Luca Di Rienzo, Renzo Bazzochi and Angelo Manara. "Circular Arrays of Magnetic Sensors for Current Measurement". IEEE Transactions on Instrumentation and Measurement, Vol. 50, $\mathrm{n}^{\mathrm{o}}$ 5, pp 1093 to 1096 . October 2001.

[4]. P. Niewczas, W. Iain Madden, W. Craig Michie, A. Cruden and J.R. McDonald. "Magnetic Crosstalk Compensation for an Optical Current Transducer". IEEE Transactions on Instrumentation and Measurement, Vol. 50, $\mathrm{n}^{\circ}$ 5, pp 1071 to 1075 . October 2001.

[5]. A. Usón, J. Letosa, F.J. Arcega, M. Samplón and J.S. Artal "Study of Measurement Errors in a Current Sensor with Air-Gap". Internacional Conference on Renewable Energy and Power Quality, ICREPQ 2005. Zaragoza, Spain. March 2005.

[6]. M. Imamura, M. Nakahara, T. Yamaguchi and S. Tamura. "Analysis of Magnetic Fields due to Threephase bus bar Current for the Design of an Optical Current Transformer". IEEE Transactions on Magnetic, Vol. 34, no 4, pp 2274 to 2279. July 1998.

[7]. J. Letosa, J. S. Artal, F. J. Arcega, M. Samplón, and A. Usón. "Modelization of Current Sensors by Finite Elements Method". Elsevier Science Ltd. Measurement, Vol 35, issue 3, pp 233 to 241. April 2004. 